\title{
Spatio-temporal Optimization of Multi-phase Movements: Dealing with Contacts and Switching Dynamics
}

\author{
Jun Nakanishi, Andreea Radulescu and Sethu Vijayakumar
}

\begin{abstract}
We address the optimal control problem of robotic systems with variable stiffness actuation (VSA) including switching dynamics and discontinuous state transitions. Our focus in this paper is to consider tasks that have multiple phases of movement, contacts and impacts with the environment. By modelling such tasks as an approximate hybrid dynamical system with time-based switching, we develop a systematic methodology to simultaneously optimize control commands, stiffness profiles and temporal aspect of the movement such as switching instances and total movement duration. Numerical evaluations on a simple switching system, a realistic brachiating robot model with VSA, and a hopper with variable stiffness springs demonstrate the effectiveness of the proposed approach.
\end{abstract}

\section{INTRODUCTION}

Towards the aim of achieving highly dynamic and flexible movements in close interaction with the environment, recently, a number of various variable stiffness actuators (VSAs) have been developed (see [1] for reviews). VSAs are composed of mechanically adjustable compliant mechanisms with the capability of simultaneous modulation of stiffness and output torque. In previous studies, benefits of variable stiffness actuation such as energy storage capability in explosive movements have been demonstrated, leading to an improvement of task performance [2], [3], and exploitation of passive dynamics in periodic movements [4], [5] and in brachiation tasks [6]. However, traditional approaches have focused on the optimal control formulation over a predetermined time horizon with smooth, continuous plant dynamics. When considering tasks that consist of multiple phases of movements including switching dynamics and discrete state transition (arising from interaction with the environment), a typical formulation with a first-exit strategy would result in a suboptimal solution.

In this paper, we investigate spatio-temporal stiffness optimization in such problems in order to exploit the benefits of variable stiffness actuation. Locomotion and juggling dynamics with intermittent contacts and impacts are often modelled as hybrid dynamical systems which consist of (multiple sets of switching) continuous dynamics and discontinuous state transition determined by switching surfaces (state based switching) [7]-[10]. From a control theoretic perspective, a significant effort has been made to address optimal control problems of a various class of hybrid systems [11]-[15]. However, illustrative examples in the control literature are confined to relatively low-dimensional and simple dynamical systems. Optimization for such hybrid dynamics is nontrivial especially in the case of state-based switching since

School of Informatics, University of Edinburgh, United Kingdom. Email: jun.nakanishi@ed.ac.uk it forms a multipoint boundary value problem with several interior-point constraints [16]. Instead of using hybrid dynamics modelling, different optimization approaches to dealing with multiple contact events have been proposed, e.g., model predictive control with smooth approximation of contract dynamics [17] and direct trajectory optimization methods by nonlinear programming [18]. In these studies, contact forces are explicitly included as constraint forces with inequality constraints, whereas in hybrid dynamics representation, contact events are simply modelled as instantaneous discrete state transition.

Here, we suggest an approximate approach to the hybrid optimal control problem, where the multiple phases of movement are modelled as a time-based switching hybrid dynamics assuming that the sequence of switching is known. In this paper, we address the following aspects:

1) nonlinear time-based switching dynamics with continuous control input

2) nonlinear discrete state transition

3) realistic plant dynamics with a VSA model

4) composite cost function to describe a task with multiphase movements

5) optimization of control command and stiffness across multiple phases

6) optimization of switching instances

7) optimization of final time (total movement duration)

To our knowledge, while there exists previous work separately addressing some of the specific points above [14], [15], [19]-[21], we have yet to find a study which has considered all these issues.

The rest of this paper is organized as follows: First, a hybrid optimal control problem with time-based switching is formulated. Next, we present an iterative solution method by extending the iterative linear quadratic regulator (iLQR) algorithm [22] in order to incorporate switching dynamics and discrete state transition. In addition, we present a temporal optimization algorithm for hybrid dynamics. The proposed algorithm provides a locally optimal feedback control law. In the evaluation, we start by showing a simple example with a linear switching system, highlighting the need for multi-phase composite optimization. Then, we consider plant dynamics with VSA including realistic actuator dynamics model with switching dynamics and discrete state transition for a brachiating robot. In addition, the proposed algorithm is applied to find an appropriate flight/stance time and optimize the hip and leg stiffness in the hopping robot with multiple phases, highlighting improved robustness and efficiency. 


\section{PROBlem Formulation}

A. Hybrid Dynamics with Time-based Switching and Discrete State Transition

Consider a class of hybrid systems

$$
\begin{aligned}
\dot{\mathbf{x}} & =\mathbf{f}_{i}(\mathbf{x}, \mathbf{u}), i \in I=\{1,2, \cdots, M\} \\
\mathbf{x}\left(T_{j}^{+}\right) & =\boldsymbol{\Delta}^{i_{j-1}, i_{j}}\left(\mathbf{x}\left(T_{j}^{-}\right)\right)
\end{aligned}
$$

where $\mathbf{f}_{i}: \mathbb{R}^{n} \times \mathbb{R}^{m} \rightarrow \mathbb{R}^{n}$ is the $i$-th subsystem, $\mathbf{x} \in \mathbb{R}^{n}$ is a state vector, $\mathbf{u} \in \mathbb{R}^{m}$ is a control input vector and $I$ is the set of indices for subsystems [15]. When the dynamics switch from subsystem $i_{j-1}$ to $i_{j}$ at $t=T_{j}$, we assume that instantaneous discrete (discontinuous) state transition is introduced, which is denoted by a map $\boldsymbol{\Delta}^{i_{j-1}, i_{j}}$ in (2) for $j=1, \cdots, K \cdot \mathbf{x}\left(T_{j}^{+}\right)$and $\mathbf{x}\left(T_{j}^{-}\right)$denote the post- and pretransition states, respectively.

When the finite number of switching is given as $K$, define the timed switching sequence $\sigma$ in $\left[T_{0}, T_{f}\right]$ as [15]

$$
\sigma=\left(\left(T_{0}, i_{0}\right),\left(T_{1}, i_{1}\right), \cdots,\left(T_{K}, i_{K}\right)\right)
$$

where $T_{0} \leq T_{1} \leq \cdots \leq T_{K} \leq T_{f}=T_{K+1}$ (i.e., monotonically increasing switching instances) and $i_{j} \in I$ for $j=0, \cdots, K$. This means that at $t=T_{j}$, subsystem switches from $i_{j-1}$ to $i_{j}$ and subsystem $i_{j}$ is active for $T_{j} \leq$ $t<T_{j+1}$. In this paper, the sequence of switching is assumed to be given, e.g., $(1,2, \cdots, K, K+1)$ or $(1,2,1,2, \cdots)$. Fig. 1 depicts a schematic diagram of a hybrid system we consider in this paper.

Note that with the definition of the switching sequence above, the hybrid dynamics we consider can be equivalently described in a more intuitive and compact representation [23]:

$$
\begin{aligned}
\dot{\mathbf{x}} & =\mathbf{f}_{i_{j}}(\mathbf{x}, \mathbf{u}), \quad T_{j} \leq t<T_{j+1} \\
\mathbf{x}\left(T_{j}^{+}\right) & =\boldsymbol{\Delta}^{i_{j-1}, i_{j}}\left(\mathbf{x}\left(T_{j}^{-}\right)\right)
\end{aligned}
$$

where $j=0, \cdots, K$ for (4) and $j=1, \cdots, K$ for (5).

\section{B. Robot Dynamics with Variable Stiffness Actuation}

Consider the robot dynamics with variable stiffness actuation

$$
\begin{gathered}
\mathbf{M}_{i}(\mathbf{q}) \ddot{\mathbf{q}}+\mathbf{C}_{i}(\mathbf{q}, \dot{\mathbf{q}}) \dot{\mathbf{q}}+\mathbf{g}_{i}(\mathbf{q})+\mathbf{D}_{i} \dot{\mathbf{q}}=\boldsymbol{\tau}_{i}\left(\mathbf{q}, \mathbf{q}_{m}\right) \\
\ddot{\mathbf{q}}_{m}+2 \boldsymbol{\alpha}_{i} \dot{\mathbf{q}}_{m}+\boldsymbol{\alpha}_{i}^{2} \mathbf{q}_{m}=\boldsymbol{\alpha}_{i}^{2} \mathbf{u}
\end{gathered}
$$

where $i$ denotes the $i$-th subsystem, $\mathbf{q}$ is the joint angle vector, $\mathbf{q}_{m}$ is the motor position vector of the VSA, $\mathbf{M}$ is the inertia matrix, $\mathbf{C}$ is the Coriolis term, $\mathbf{g}$ is the gravity vector, $\mathbf{D}$ is the viscous damping matrix, and $\tau$ are the joint torques from the variable stiffness mechanism. (6) denotes the rigid body dynamics of the robot and (7) denotes the servo motor dynamics in the variable stiffness actuator. In (7), $\alpha$ determines the bandwidth of the servo motors and $\mathbf{u}$ is the motor position command [2]. We assume that the range of control command $\mathbf{u}$ is limited between $\mathbf{u}_{\min }$ and $\mathbf{u}_{\max }$.

In this paper, we consider a VSA model in which the joint torques are given in the form

$$
\boldsymbol{\tau}\left(\mathbf{q}, \mathbf{q}_{m}\right)=\mathbf{A}^{T}\left(\mathbf{q}, \mathbf{q}_{m}\right) \mathbf{F}\left(\mathbf{q}, \mathbf{q}_{m}\right)
$$

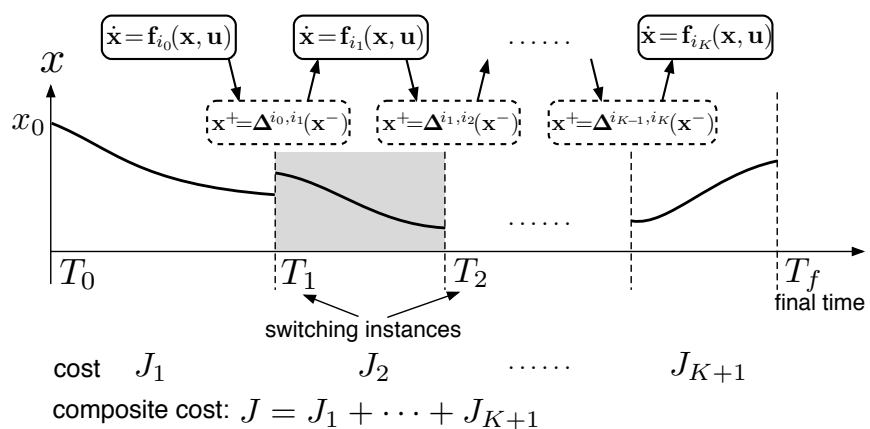

Fig. 1. A hybrid system with time-based switching dynamics and discrete state transition with a known sequence. The objective is to find an optimal control command $\mathbf{u}$, switching instances $T_{i}$ and final time $T_{f}$ which minimizes the composite cost $J=J_{1}+\cdots+J_{K+1}$.

where $\mathbf{A}$ is the moment arm matrix and $\mathbf{F}$ is the forces by the elastic elements [2] and the joint stiffness is defined as

$$
\mathbf{K}=-\frac{\partial \boldsymbol{\tau}}{\partial \mathbf{q}} \text {. }
$$

In the state space representation (1), the plant dynamics are formulated as

$$
\dot{\mathbf{x}}=\mathbf{f}_{i}(\mathbf{x}, \mathbf{u})
$$

where

$$
\mathbf{f}_{i}=\left[\begin{array}{c}
\mathbf{x}_{2} \\
\mathbf{M}_{i}^{-1}\left(\mathbf{x}_{1}\right)\left(-\mathbf{C}_{i}\left(\mathbf{x}_{1}, \mathbf{x}_{2}\right) \mathbf{x}_{2}-\mathbf{g}_{i}\left(\mathbf{x}_{1}\right)-\mathbf{D}_{i} \dot{\mathbf{x}}_{2}+\boldsymbol{\tau}_{i}\left(\mathbf{x}_{1}, \mathbf{x}_{3}\right)\right) \\
\mathbf{x}_{4} \\
-\boldsymbol{\alpha}_{i}^{2} \mathbf{x}_{3}-2 \boldsymbol{\alpha}_{i} \mathbf{x}_{4}+\boldsymbol{\alpha}_{i}^{2} \mathbf{u}
\end{array}\right]
$$

and $\mathbf{x}=\left[\mathbf{x}_{1}^{T}, \mathbf{x}_{2}^{T}, \mathbf{x}_{3}^{T}, \mathbf{x}_{4}^{T}\right]^{T}=\left[\mathbf{q}^{T}, \dot{\mathbf{q}}^{T}, \mathbf{q}_{m}^{T}, \dot{\mathbf{q}}_{m}^{T}\right]^{T}$.

\section{Movement Optimization of Multiple Phases}

We consider the composite cost function

$$
J=\phi\left(\mathbf{x}\left(T_{f}\right)\right)+\sum_{j=1}^{K} \psi^{j}\left(\mathbf{x}\left(T_{j}^{-}\right)\right)+\int_{T_{0}}^{T_{f}} h(\mathbf{x}, \mathbf{u}) d t
$$

to describe the full movement with multiple phases, where $\phi\left(\mathbf{x}\left(T_{f}\right)\right)$ is the terminal cost, $\psi^{j}\left(\mathbf{x}\left(T_{j}^{-}\right)\right)$is the via-point cost at the $j$-th switching instance and $h(\mathbf{x}, \mathbf{u})$ is the running cost.

In our previous work [6], we have optimized each cost function $J_{i}$ for each phase separately in a sequential manner to optimize multiple sequence of swing locomotion in a robot brachiation example, where

$$
J_{j}=\psi^{j}\left(\mathbf{x}\left(T_{j}^{-}\right)\right)+\int_{T_{j-1}}^{T_{j}} h(\mathbf{x}, \mathbf{u}) d t \quad \text { for } j=1, \cdots, K
$$

and

$$
J_{K+1}=\phi\left(\mathbf{x}\left(T_{f}\right)\right)+\int_{T_{K}}^{T_{f}} h(\mathbf{x}, \mathbf{u}) d t
$$

using the terminal state for the $j$-th sequence as the initial condition for the $(j+1)$-th sequence with the discrete state transition (2). In this case, each cost function can be (locally) optimized, however, the total cost $J=\sum_{j=1}^{K+1} J_{j}$ may be suboptimal.

For given plant dynamics (4) and state transition (5), the optimization problem we consider is to a) find an optimal 
feedback control law $\mathbf{u}=\mathbf{u}(\mathbf{x}, t)$ which minimizes the composite cost (12) and b) simultaneously optimize switching instances $T_{1}, \cdots, T_{k}$ and the final time $T_{f}$ as well. Note that in previous studies on optimization of switching instances, the final time is fixed.

\section{SPATIO-TEMPORAL OPTIMIZATION WITH DisCONTINUOUS STATE TRANSITIONS}

In this section, first we extend iLQR - an approximate optimal feedback control (OFC) solver (similar arguments apply for the stochastic equivalent iLQG [22]) in order to incorporate timed switching dynamics with discrete and discontinuous state transitions. Then, we present a temporal optimization algorithm to optimize the switching instances and the total movement duration.

\section{A. Optimal Control of Switching Dynamics and Discrete} State Transition

In brief, the iLQR method solves an optimal control problem of the locally linear quadratic approximation of the nonlinear dynamics and the cost function around a nominal trajectory $\overline{\mathbf{x}}$ and control sequence $\overline{\mathbf{u}}$ in discrete time, and iteratively improves the solutions.

In order to incorporate switching dynamics and discrete state transition with a given switching sequence $\sigma$ in (3), the hybrid dynamics (1) and (2) are linearized in discrete time around the nominal trajectory and control sequence as

$$
\begin{aligned}
\delta \mathbf{x}_{k+1} & =\mathbf{A}_{k} \delta \mathbf{x}_{k}+\mathbf{B}_{k} \delta \mathbf{u}_{k} \\
\delta \mathbf{x}_{k_{j}}^{+} & =\boldsymbol{\Gamma}_{k_{j}} \delta \mathbf{x}_{k_{j}}^{-} \\
\mathbf{A}_{k} & =\mathbf{I}+\left.\Delta t_{j} \frac{\partial \mathbf{f}_{i_{j}}}{\partial \mathbf{x}}\right|_{\mathbf{x}=\mathbf{x}_{k}}, \quad \mathbf{B}_{k}=\left.\Delta t_{j} \frac{\partial \mathbf{f}_{i_{j}}}{\partial \mathbf{u}}\right|_{\mathbf{u}=\mathbf{u}_{k}} \\
\boldsymbol{\Gamma}_{k_{j}} & =\left.\frac{\partial \boldsymbol{\Delta}^{i_{j-1}, i_{j}}}{\partial \mathbf{x}}\right|_{\mathbf{x}=\mathbf{x}_{k_{j}}^{-}}
\end{aligned}
$$

where $\delta \mathbf{x}_{k}=\mathbf{x}_{k}-\overline{\mathbf{x}}_{k}, \delta \mathbf{u}_{k}=\mathbf{u}_{k}-\overline{\mathbf{u}}_{k}, k$ is the discrete time step, $\Delta t_{j}$ is the sampling time for the time interval $T_{j} \leq t<T_{j+1}$, and $k_{j}$ is the $j$-th switching instance in the discretized time step.

The composite cost function (12) is locally approximated in a quadratic form as

$$
\begin{aligned}
\Delta J & =\delta \mathbf{x}_{N}^{T} \phi_{\mathbf{x}}+\frac{1}{2} \delta \mathbf{x}_{N}^{T} \phi_{\mathbf{x} \mathbf{x}} \delta \mathbf{x}_{N} \\
& +\sum_{j=1}^{K}\left(\left(\delta \mathbf{x}_{k_{j}}^{-}\right)^{T} \psi_{\mathbf{x}}^{j}+\frac{1}{2}\left(\delta \mathbf{x}_{k_{j}}^{-}\right)^{T} \psi_{\mathbf{x} \mathbf{x}}^{j} \delta \mathbf{x}_{k_{j}^{-}}\right) \\
& +\sum_{k=1}^{N}\left(\delta \mathbf{x}_{k}^{T} h_{\mathbf{x}}+\delta \mathbf{u}_{k}^{T} h_{\mathbf{u}}\right. \\
& \left.+\frac{1}{2} \delta \mathbf{x}_{k}^{T} h_{\mathbf{x} \mathbf{x}} \delta \mathbf{x}_{k}+\frac{1}{2} \delta \mathbf{u}_{k}^{T} h_{\mathbf{u u}} \delta \mathbf{u}_{k}+\delta \mathbf{u}_{k} h_{\mathbf{u x}} \delta \mathbf{x}_{k}\right) \Delta t_{j}
\end{aligned}
$$

and a local approximation of the optimal cost-to-go function is defined as

$$
v_{k}\left(\delta \mathbf{x}_{k}\right)=\frac{1}{2} \delta \mathbf{x}_{k}^{T} \mathbf{S}_{k} \delta \mathbf{x}_{k}+\delta \mathbf{x}_{k}^{T} \mathbf{s}_{k}
$$

For the dynamics (15), the cost-to-go parameters in (20), $\mathbf{S}_{k}, \mathbf{s}_{k}$ are updated with a modified Riccati equations ${ }^{1}$ (see

\footnotetext{
${ }^{1}$ At the final time $k=N, \mathbf{S}_{N}=\phi_{\mathbf{x x}}$ and $\mathbf{s}_{N}=\phi_{\mathbf{x}}$.
}

$\overline{\text { Algorithm } 1 \text { Complete optimization algorithm for hybrid }}$ dynamics with temporal optimization

1: Input:

- Timed switching plant dynamics $\mathbf{f}_{i}(1)$, discrete state transition $\boldsymbol{\Delta}^{i_{j-1}, i_{j}}(2)$ and switching sequence $\sigma$ (3)

- Composite cost function $J(12)$

2: Initialize:

- Nominal switching instance and final time $T_{1}, \cdots, T_{K}$ and $T_{f}$ and corresponding discrete sampling time $\Delta t_{0}, \cdots, \Delta t_{K}$ as in (29) and (30)

- Nominal control sequence $\overline{\mathbf{u}}$ and corresponding $\overline{\mathbf{x}}$

3: repeat

4: repeat

5: Optimize control sequence $\overline{\mathbf{u}}$ :

- $\quad$ Obtain linearized time-based switching dynamics (15) and state transition (16) around $\overline{\mathbf{x}}$ and $\overline{\mathbf{u}}$ in discrete time with current $\Delta t_{j}$

- Compute quadratic approximation of the composite cost (19)

- $\quad$ Solve local optimal control problem to obtain $\delta \mathbf{u}$ (21)

- $\quad$ Apply $\delta \mathbf{u}$ to the linearized hybrid dynamics (15) and (19)

- Update nominal control sequence $\overline{\mathbf{u}} \leftarrow \overline{\mathbf{u}}+\delta \mathbf{u}$, trajectory $\overline{\mathbf{x}}$ and cost $J$

6: until convergence

7: Temporal optimization: update $\Delta t_{j}$ :

- $\quad$ Update the vector of temporal scaling factor $\boldsymbol{\beta}$ and corresponding sampling time $\Delta t_{0}, \cdots, \Delta t_{K}$ in (27) via gradient descent.

- $\quad$ Obtain corresponding switching instances $T_{j}$ (29) and final time $T_{f}(30)$

8: until convergence

9: Output:

- Optimal feedback control law $\mathbf{u}(\mathbf{x}, t)$ : feedforward optimal control sequence $\mathbf{u}_{o p t}$, optimal trajectory $\mathbf{x}_{o p t}(t)$ and optimal gain matrix $\mathbf{L}_{o p t}(t)$ :

$\mathbf{u}(\mathbf{x}, t)=\mathbf{u}_{\text {opt }}(t)+\mathbf{L}_{\text {opt }}(t)\left(\mathbf{x}(t)-\mathbf{x}_{\text {opt }}(t)\right)$

- Optimal switching instance $T_{1}, \cdots, T_{K}$ and final time $T_{f}$

- Optimal composite cost $J$

[22] for details) and the local control law $\delta \mathbf{u}_{k}$ of the form

$$
\delta \mathbf{u}_{k}=\mathbf{l}_{k}+\mathbf{L}_{k} \delta \mathbf{x}_{k}
$$

is obtained from the Bellman equation

$$
v_{k}\left(\delta \mathbf{x}_{k}\right)=\min _{\delta \mathbf{u}}\left\{h_{k}\left(\delta \mathbf{x}_{k}, \delta \mathbf{u}_{k}\right)+v_{k+1}\left(\delta \mathbf{x}_{k+1}\right)\right\}
$$

by substituting (15) and (20) into the equation (22), where $h_{k}$ is the local approximation of the running cost in (19). At the instance of discrete state transition $k=k_{j}$, the following cost-to-go parameter update is added (cf. [14], [15]):

$$
\begin{aligned}
\mathbf{S}_{k_{j}}^{-} & =\psi_{\mathbf{x x}}^{j}+\boldsymbol{\Gamma}_{k_{j}}^{T} \mathbf{S}_{k_{j}}^{+} \boldsymbol{\Gamma}_{k_{j}} \\
\mathbf{s}_{k_{j}}^{-} & =\psi_{\mathbf{x}}^{j}+\boldsymbol{\Gamma}_{k_{j}}^{T} \mathbf{s}_{k_{j}}^{+}
\end{aligned}
$$

which is derived from the Bellman equation

$$
v_{k_{j}}\left(\delta \mathbf{x}_{k_{j}}^{-}\right)=\psi_{k_{j}}^{j}\left(\delta \mathbf{x}_{k_{j}}^{-}\right)+v_{k_{j}}\left(\delta \mathbf{x}_{k_{j}}^{+}\right)
$$



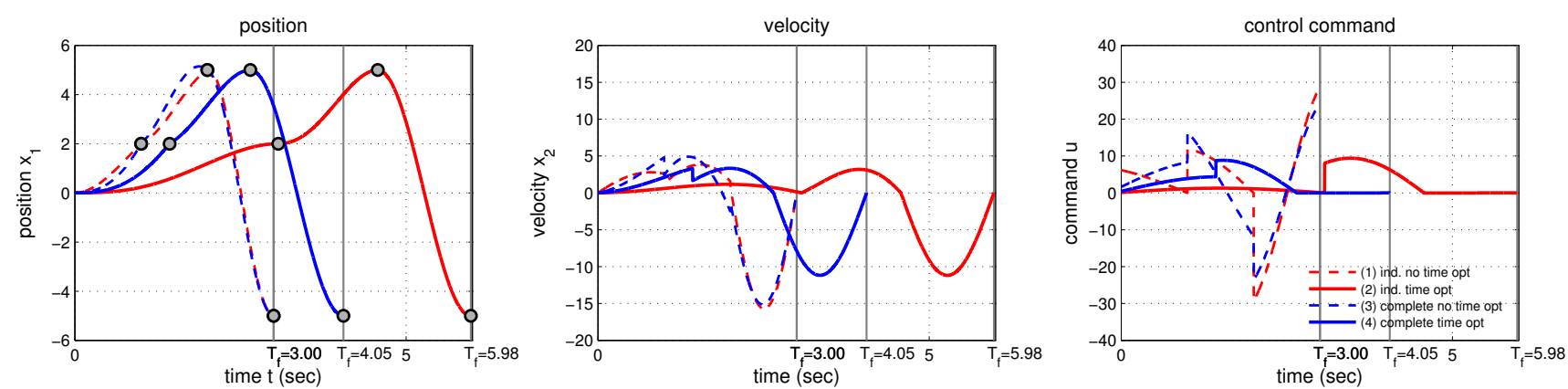

Fig. 2. Optimization results of the linear switching system example. Left: position $x_{1}$, via-points and final target are marked in circles. Center: velocity $x_{2}$. Right: control command $u$.

at $k=k_{j}$, where $\psi_{k_{j}}^{j}\left(\delta \mathbf{x}_{k_{j}}^{-}\right)$is the local approximation of the via-point cost (19).

Once we have a locally optimal control command $\delta \mathbf{u}$, the nominal control sequence is updated as $\overline{\mathbf{u}} \leftarrow \overline{\mathbf{u}}+\delta \mathbf{u}$. Then, the new nominal trajectory $\overline{\mathrm{x}}$ is computed by running the obtained control $\overline{\mathbf{u}}$ and the above process is iterated until convergence.

\section{B. Temporal Optimization}

In our prior work [24], we proposed a temporal optimization algorithm to simultaneously optimize the control commands and temporal parameters by introducing a mapping $\beta(t)$ from the real time $t$ to a canonical time $t^{\prime}$ as

$$
t^{\prime}=\int_{0}^{t} \frac{1}{\beta(s)} d s
$$

where $\beta(t)$ is then optimized to scale the temporal aspect of the movement. We have applied this [4], [6] to optimize the frequency of the periodic movement and the movement duration of swing locomotion in a brachiation task. In these problems, we discretized (26) with an assumption that $\beta(t)$ and $\Delta t$ are constant throughout the movement as $\Delta t^{\prime}=$ $\frac{1}{\beta} \Delta t$. In order to optimize the switching instances and the total movement duration, in this work, we introduce a scaling parameter and sampling time for each duration between switching as (cf. (17) and (19))

$$
\Delta t_{j}^{\prime}=\frac{1}{\beta_{j}} \Delta t_{j} \quad \text { for } \quad T_{j} \leq t<T_{j+1}
$$

where $j=0, \cdots, K$. By optimizing the vector of temporal scaling factors $\boldsymbol{\beta}=\left[\beta_{0}, \cdots, \beta_{K}\right]^{T}$ via gradient descent

$$
\boldsymbol{\beta} \leftarrow \boldsymbol{\beta}-\eta \nabla_{\beta} J
$$

where $\eta>0$ is a learning rate, we can obtain each switching instance

$$
T_{j+1}=\left(k_{j+1}-k_{j}\right) \Delta t_{j}^{\prime}+T_{j}
$$

and total movement duration

$$
T_{f}=\sum_{j=0}^{K}\left(k_{j+1}-k_{j}\right) \Delta t_{j}^{\prime}+T_{0}
$$

where $k_{0}=1$ and $k_{K+1}=N$. In the complete optimization, computation of optimal feedback control law and temporal scaling parameter update are iteratively performed until convergence in an EM-like (expectation-maximization) manner. A pseudocode of the complete algorithm is summarized in Algorithm 1.
TABLE I

COMPARISON OF THE RESULTS OF THE LINEAR SWITCHING SYSTEM.

\begin{tabular}{llc}
\hline & $\left(T_{1}, T_{2}, T_{f}\right)(\mathrm{sec})$ & cost $J$ \\
\hline (1) ind. no time opt. & $(1,2,3)$ & 41.7 \\
\hline (2) ind. time opt. & $(3.07,4.57,5.98)$ & 8.31 \\
\hline (3) complete, no time opt. & $(1,2,3)$ & 34.4 \\
\hline (4) complete, time opt. & $(1.43,2.65,4.05)$ & $\mathbf{6 . 5 9}$ \\
\hline
\end{tabular}

\section{Evaluations ${ }^{2}$}

\section{A. Linear Switching System}

In order to illustrate the effectiveness of the proposed approach, first, we consider the following example of a hybrid linear system with linear discrete state transition:

$$
\begin{aligned}
\dot{\mathbf{x}} & =\mathbf{A}_{i} \mathbf{x}+\mathbf{B}_{i} \mathbf{u} \\
\mathbf{x}^{+} & =\mathbf{\Gamma} \mathbf{x}^{-}
\end{aligned}
$$

where $\mathbf{x}=\left[x_{1}, x_{2}\right]^{T}, \mathbf{A}_{i}=\left[\begin{array}{cc}0 & 1 \\ -k_{i} & 0\end{array}\right], \mathbf{B}_{i}=\left[\begin{array}{l}0 \\ 1\end{array}\right]$, $k_{1}=1, k_{2}=2, k_{3}=5$ and $\boldsymbol{\Gamma}=\left[\begin{array}{cc}1 & 0 \\ 0 & 0.5\end{array}\right]$. The subsystem switches with discrete state transitions from $i=1$ to $i=2$ at $t=T_{1}$ and from $i=2$ to $i=3$ at $t=T_{2}$. The cost function to be minimized is

$$
\begin{aligned}
J & =\left(\mathbf{x}\left(T_{f}\right)-\mathbf{x}_{f}^{*}\right)^{T} \mathbf{Q}_{T_{f}}\left(\mathbf{x}\left(T_{f}\right)-\mathbf{x}_{f}^{*}\right) \\
& +\sum_{j=1}^{2} Q_{T_{j}}\left(x_{1}\left(T_{j}^{-}\right)-x_{1, j}^{*}\right)^{2}+\int_{0}^{T_{f}} R u^{2} d t
\end{aligned}
$$

where $\mathbf{x}_{f}^{*}=[-5,0]^{T}$ is the desired position and velocity at $t=T_{f}, x_{1,1}^{*}=2$ and $x_{1,2}^{*}=5$ are the desired positions at the via-points. The initial states are chosen as $\mathbf{x}_{0}=[0,0]^{T}$. The weights in the cost are given as $\mathbf{Q}_{T_{f}}=\operatorname{diag}(500,500), Q_{T_{j}}=500$ and $R=0.1$. Note that at the via-points, the velocity is not penalized, but at the end point, both the position and the velocity are specified. We find an optimal control command $u$, switching instances $T_{k}$ and the movement duration $T_{f}$. The initial switching times and movement duration are chosen as $T_{1}=1, T_{2}=2$ and $T_{f}=3$.

Table I summarizes the comparison among the following four cases:

1) sequential individual movement optimization without temporal optimization

2) sequential individual movement optimization with temporal optimization

\footnotetext{
${ }^{2} \mathrm{~A}$ video clip highliting the results is available at http: / / youtu.be/dUhL36b4eWg
} 


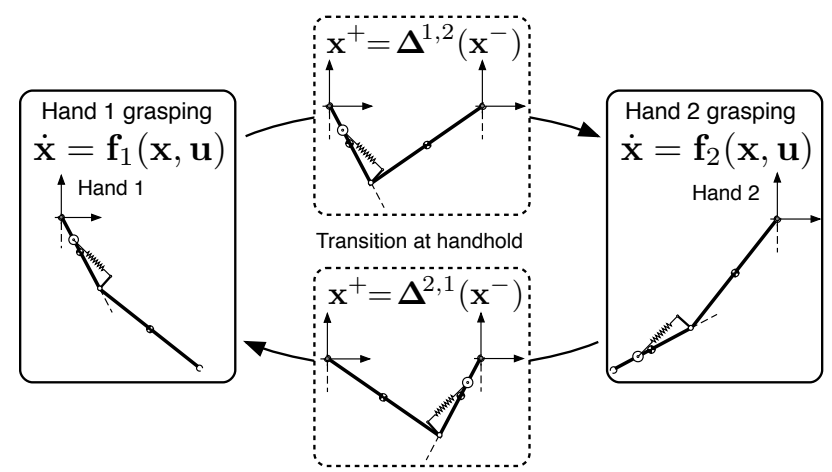

Fig. 3. Hybrid dynamics modelling of a brachiating robot with asymmetric structure. The dynamics switches at the transition from a handhold to the next with a discrete state mapping.

3) complete movement optimization without temporal optimization

4) complete movement optimization with temporal optimization

In the sequential individual movement optimization, each segment of the movement to the via-point is individually optimized in a sequential manner followed by the next movement starting from the end-point of the previous movement. In the complete movement optimization, the entire movement over multiple via-points including switching dynamics and discrete state transition is optimized from the initial state to the end target. Fig. 2 shows the optimized trajectories and control command. The results in Table I implies that sequential optimization resulted in a sub-optimal solution with a higher cost compared to complete movement optimization. Furthermore, when temporal optimization is employed, the cost is significantly reduced, which suggests the effectiveness of the proposed approach.

\section{B. Spatio-temporal Optimization of Multiple Swings in Robot Brachiation}

In this section, we evaluate the effectiveness of the proposed approach in robot brachiation that incorporates switching dynamics and multiple phases of the movement in a realistic VSA actuator model. Brachiation is a form of swing locomotion from handhold to handhold. For successful task execution by exploiting the benefits of VSA and passive dynamics, the spatio-temporal aspect of joint stiffness and control command needs to be optimized.

1) Robot Dynamics with Variable Stiffness Actuator Model: We consider a two-link underactuated brachiating robot with a MACCEPA variable stiffness actuator model [6]. The robot dynamics (6) is given by

$$
\mathbf{M}_{i}(\mathbf{q}) \ddot{\mathbf{q}}+\mathbf{C}_{i}(\mathbf{q}, \dot{\mathbf{q}}) \dot{\mathbf{q}}+\mathbf{g}_{i}(\mathbf{q})+\mathbf{D}_{i} \dot{\mathbf{q}}=\left[\begin{array}{c}
0 \\
\tau\left(\mathbf{q}, \mathbf{q}_{m}\right)
\end{array}\right]
$$

where only the second joint has actuation and $\mathbf{q}=$ $\left[q_{1}, q_{2}\right]^{T}$. The joint torque with the MACCEPA model [25] in (8) is given by

$$
\tau=\frac{B C \sin \left(q_{m 1}-q_{2}\right)}{\gamma} F
$$

where $\mathbf{q}_{m}=\left[q_{m 1}, q_{m 2}\right]^{T}$ are the servo motor positions in the VSA model, $F$ is the spring tension

$$
F=\kappa\left(l-l_{0}\right)
$$

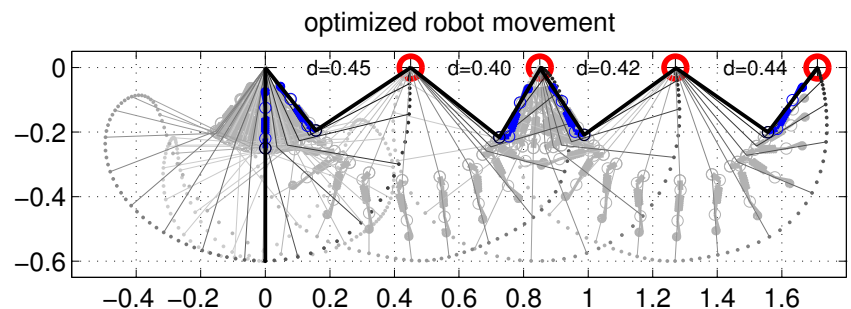

(a) Optimized movement of the robot
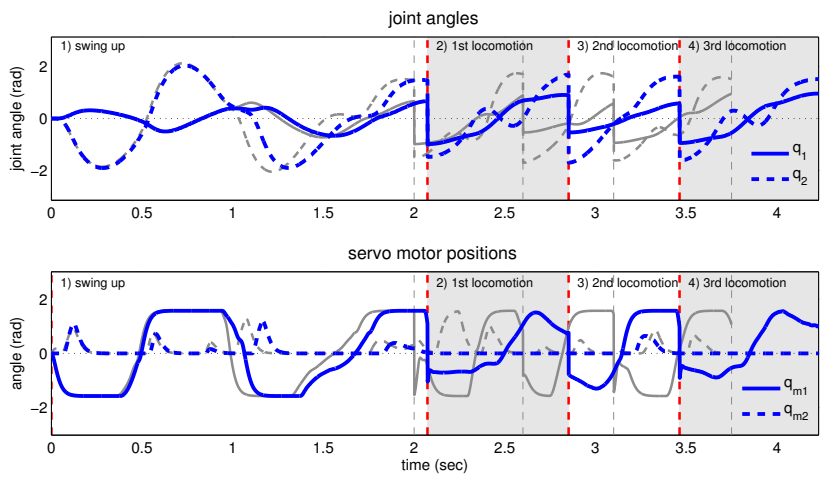

(b) Joint trajectories and servo motor positions

Fig. 4. (a) Optimized movement of the robot. (b) Comparison of joint trajectories and servo motor positions between complete optimization with temporal optimization and individual movement optimization without temporal optimization. Thick blue lines: complete optimization with temporal optimization. Thin gray lines: individual optimization without temporal optimization.

$\gamma=\sqrt{B^{2}+C^{2}-2 B C \cos \left(q_{m 1}-q_{2}\right)}$ and $l=\gamma+r_{d} q_{m 2}$. We assume that the robot has a generic asymmetric structure in the dynamics (unequal mass and/or link length). Hence, the effective model switches between $i=1$ and $i=2$ interchangeably at the switching instance $T_{k}$ when the robot grasps the bar as $\sigma=\left(\left(T_{0}, 1\right),\left(T_{1}, 2\right),\left(T_{2}, 1\right), \ldots\right)$ (see Fig. 3).

To formulate optimization, we use the state space representation in (11). At the transition at handhold, an affine discrete state transition $\mathbf{x}^{+}=\boldsymbol{\Delta}\left(\mathbf{x}^{-}\right)=\Gamma \mathbf{x}^{-}+\gamma$ is introduced to shift the coordinate system for the next handhold and reset the joint velocities of the robot to zero, which is defined as

$$
\boldsymbol{\Gamma}=\operatorname{diag}\left(\boldsymbol{\Gamma}_{1}, \cdots, \boldsymbol{\Gamma}_{4}\right)
$$

where

$$
\boldsymbol{\Gamma}_{1}=\left[\begin{array}{cc}
1 & 1 \\
0 & -1
\end{array}\right], \boldsymbol{\Gamma}_{2}=\left[\begin{array}{ll}
0 & 0 \\
0 & 0
\end{array}\right], \boldsymbol{\Gamma}_{3}=\boldsymbol{\Gamma}_{4}=\left[\begin{array}{cc}
-1 & 0 \\
0 & 1
\end{array}\right]
$$

and $\gamma=[-\pi, 0, \cdots, 0]^{T}$. Note that in this example, we have $\boldsymbol{\Delta}=\boldsymbol{\Delta}^{1,2}=\boldsymbol{\Delta}^{2,1}$.

2) Optimization Results: We consider a brachiating task with multiple phases of the movement as follows: First, the robot swings up from the suspended posture to the target at $d_{1}=0.45(\mathrm{~m})$ and subsequently moves to the target located at $d_{2}=0.4, d_{3}=0.42$ and $d_{4}=0.44$. The composite cost function to encode this task is given by

$$
\begin{aligned}
J= & \left(\mathbf{y}\left(T_{f}\right)-\mathbf{y}_{f}^{*}\right)^{T} \mathbf{Q}_{T_{f}}\left(\mathbf{y}\left(T_{f}\right)-\mathbf{y}_{f}^{*}\right) \\
& +\sum_{j=1}^{3}\left(\mathbf{y}\left(T_{j}^{-}\right)-\mathbf{y}_{j}^{*}\right)^{T} \mathbf{Q}_{T_{j}}\left(\mathbf{y}\left(T_{j}^{-}\right)-\mathbf{y}_{j}^{*}\right) \\
& +\int_{0}^{T}\left(\mathbf{u}^{T} \mathbf{R}_{1} \mathbf{u}+R_{2} F^{2}\right) d t+w_{T} T_{1}
\end{aligned}
$$




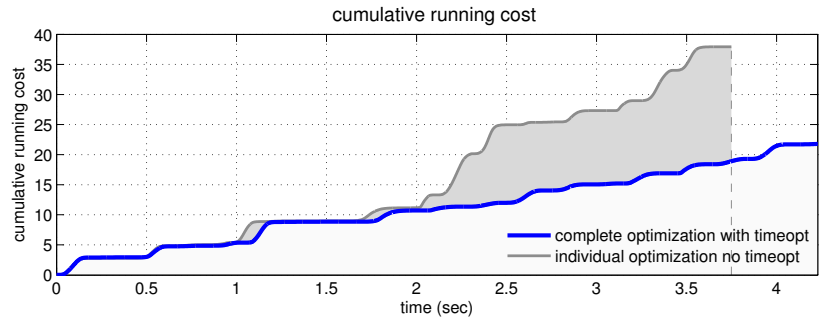

Fig. 5. Comparison of the cumulative running cost between complete optimization with temporal optimization (blue line) and individual movement optimization without temporal optimization (gray line) as a measure of control effort required to complete the task.

where $\mathbf{y}=[\mathbf{r}, \dot{\mathbf{r}}]^{T} \in \mathbb{R}^{4}$ are the position and the velocity of the gripper in the Cartesian coordinates measured from the origin at current handhold, $\mathbf{y}^{*}$ is the target values when reaching the target $\mathbf{y}^{*}=\left[\begin{array}{rl}\mathbf{r}^{*}, & \mathbf{0}\end{array}\right]^{T}$ and $F$ is the spring tension in the VSA. Note that this cost function includes the time cost $w_{T} T_{1}$ for the swing up maneuver. The term in the running cost $\mathbf{u}_{1}^{T} \mathbf{R}_{1} \mathbf{u}_{1}$ is added for regularization with a small choice of $\mathbf{R}_{1}$.

Fig. 4 (a) shows the sequence of the robot movement optimized by the proposed algorithm including temporal optimization. The optimized switching instance and total movement duration are $T_{1}=2.073, T_{2}=2.852, T_{3}=$ 3.463 and $T_{f}=4.230$ ( $\mathrm{sec}$ ). Fig. 4 (b) shows comparison between complete movement optimization with temporal optimization (thick blue lines) and sequential individual movement optimization without temporal optimization (thin gray lines). Note that at the instance of switching denoted by vertical lines, discrete state transition can be observed in these trajectories due to the definition of the coordinate transformation. The cost with the complete optimization with temporal optimization is $J=33.10$ while the cost with individual optimization without temporal optimization $\left(T_{1}=\right.$ $2.0, T_{2}=2.6, T_{3}=3.1$ and $T_{f}=3.75$ are fixed) is $J=$ 94.65. Fig. 5 shows the cumulative running cost for these two cases as a measure of the control effort required to complete the task. The result demonstrates that the proposed method is able to find optimal control commands and temporal aspect of the movement resulting in lower cost.

\section{Hopping Robot Dynamics with VSA}

In this section, we present our results on a simplified model of a one-legged hopping robot with variable stiffness actuators. We augment the hopping robot model in [27] with variable compliance elements in the hip and the leg actuators. In this hopping example, we demonstrate the feasibility of the proposed approach on an increasingly challenging task that includes switching of different mode of dynamics (flight and stance) and more complex discontinuous state transition arising from impact at touch-down. Additional difficulty in this task is to find the flight and stance time for successful task execution which is highly restricted by the underactuated nature of the intrinsic dynamics and the desired task specifications.

The objective of optimization is to find appropriate leg and hip stiffness to exploit the passive dynamics and also the required flight and stance time during one locomotion cycle in a periodic movement based on a time-based switching approximation. The obtained controller is then

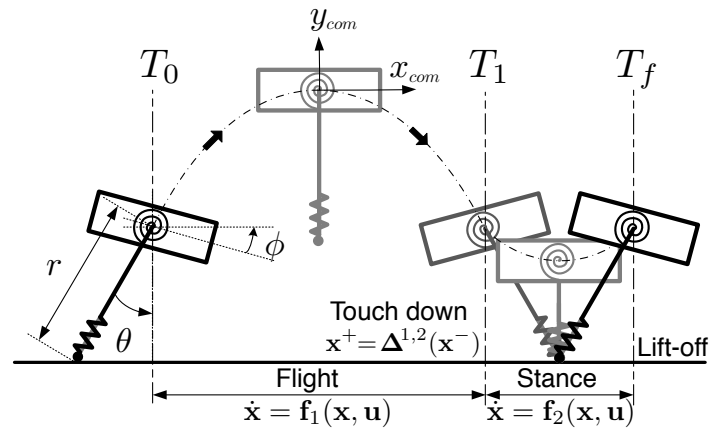

Fig. 6. Hybrid dynamics of a hopping robot model and locomotion phases during one cycle

TABLE II

COMMANDS FOR THE HOPPING ROBOT MODEL

\begin{tabular}{|c|c|c|}
\hline Command & Meaning & Unit \\
\hline$u_{1}$ & torque applied at the hip & $\mathrm{Nm}$ \\
$u_{2}$ & force applied in the leg & $\mathrm{N}$ \\
$u_{3}$ & hip joint stiffness & $\mathrm{Nm} / \mathrm{rad}$ \\
$u_{4}$ & leg stiffness & $\mathrm{N} / \mathrm{m}$ \\
\hline
\end{tabular}

applied to achieve multiple hopping cycles of locomotion on event based switching dynamics. Robustness of the obtained optimal feedback controller will be evaluated by applying external disturbances during the multiple hopping cycles to demonstrate the feasibility of the optimized controller.

1) Dynamics model of a hopping robot: The hopping dynamics switch between the flight phase and stance phase (with $i=1$ : flight and $i=2$ : stance) at the switching instance $T_{j}$ when either touch-down or lift-off conditions are met. The configuration vector of the system is defined as $\mathbf{q}=\left[x_{\text {com }}, y_{\text {com }}, \theta, \phi, r\right]^{T}$ (see Fig. 6). The general form of the flight and stance dynamics can be given by

$$
\mathbf{M}_{i} \ddot{\mathbf{q}}_{i}+\mathbf{C}_{i}\left(\mathbf{q}_{i}, \dot{\mathbf{q}}_{i}\right) \dot{\mathbf{q}}_{i}+\mathbf{g}_{i}\left(\mathbf{q}_{i}\right)=\boldsymbol{\tau}_{i}\left(\mathbf{q}_{i}, \mathbf{u}_{i}\right)
$$

where $\mathbf{q}_{1}=\left[x_{\text {com }}, y_{\text {com }}, \theta, \phi\right]^{T}$ and $\mathbf{q}_{2}=[\theta, \phi, r]^{T}$ are the partial configuration vector for the flight and stance phase, respectively (for the specific form of the dynamics in each phase, please refer to [26], [27]). $\boldsymbol{\tau}_{1}(\mathbf{q}, \mathbf{u})=$ $\left[0,0,-\tau_{\text {hip }}, \tau_{\text {hip }}\right]^{T}$ and $\boldsymbol{\tau}_{2}(\mathbf{q}, \mathbf{u})=\left[-\tau_{\text {hip }}, \tau_{\text {hip }}, \tau_{\text {leg }}\right]^{T}$ are the VSA torque/force applied to each degree of freedom where

$$
\begin{aligned}
& \tau_{\text {hip }}(\mathbf{q}, \mathbf{u})=u_{1}-u_{3}(\phi-\theta) \\
& \tau_{\text {leg }}(\mathbf{q}, \mathbf{u})=u_{2}-u_{4}\left(r-r_{0}\right)
\end{aligned}
$$

and $\mathbf{u}=\left[u_{1}, u_{2}, u_{3}, u_{4}\right]^{T}$ is the control command vector defined as in Table II. The range of the control and stiffness is limited as $u_{i, \min } \leq u_{i} \leq u_{i, \max }$.

For the purpose of optimization, the dynamics will be formulated in a state space representation of the form of $\dot{\mathbf{x}}=\mathbf{f}_{i}(\mathbf{x}, \mathbf{u})$ as in (10) with the full state vector $\mathbf{x}=$ $\left[\mathbf{q}^{T}, \dot{\mathbf{q}}^{T}\right]^{T}$ and $\mathbf{q}=\left[x_{\text {com }}, y_{\text {com }}, \theta, \phi, r\right]^{T}$. Note that in this hopping robot, we consider a simplified parallel elastic VSA model with direct force/torque and stiffness control, which does not include the motor dynamics (7).

The discontinuous impact map at touch-down is defined as

$$
\mathbf{x}^{+}=\boldsymbol{\Delta}^{1,2}\left(\mathbf{x}^{-}\right)=\left[\begin{array}{cc}
\mathbf{I} & \mathbf{0} \\
\mathbf{0} & \boldsymbol{\Lambda}(\mathbf{q})
\end{array}\right] \mathbf{x}^{-}
$$

where $\mathbf{x}=\left[\mathbf{q}^{T}, \dot{\mathbf{q}}^{T}\right]^{T}, \mathbf{q}^{+}=\mathbf{q}^{-}$and $\dot{\mathbf{q}}^{+}=\boldsymbol{\Lambda}(\mathbf{q}) \dot{\mathbf{q}}^{-}$. The matrix $\boldsymbol{\Lambda}(\mathbf{q})$ is the transition map between the pre-impact to 
post-impact velocities based on a rigid body collision model [8], [9]. The specific form of the velocity transition map is given in the study of passive running with an additional analysis of energy dissipation at impact [27]. At lift-off, the velocity of the leg is reset to zero as $\dot{r}=0$ at $r=r_{0}$.

2) Design of Composite Cost Function: In this paper, we consider a task of achieving periodic movement of continuous hopping which is a repetition of one hopping cycle while exploiting the passive dynamics and the benefits of stiffness modulation. For this purpose, first, we design a composite cost function for one hopping cycle including both the flight and stance phases and the desirable touchdown condition. Then, the obtained controller is applied to achieve multiple cycles.

Consider the following cost function:

$J=\left(\mathbf{x}\left(T_{f}\right)-\mathbf{x}_{0}\right)^{T} \mathbf{Q}_{T_{f}}\left(\mathbf{x}\left(T_{f}\right)-\mathbf{x}_{0}\right)+\Psi\left(\mathbf{x}\left(T_{1}^{-}\right)\right)+\int_{0}^{T_{f}} \mathbf{u}^{T} \mathbf{R u} d t$

where $\mathbf{Q}_{T_{f}}$ is a positive semi-definite matrix and $\mathbf{R}$ is a positive definite matrix. The purpose of the first term is to achieve periodicity of the trajectory where $\mathbf{x}_{0}$ denotes the initial state. The second term consists of two criteria:

$$
\Psi\left(\mathbf{x}\left(T_{1}^{-}\right)\right)=Q_{T_{1}, 1}\left(y_{\text {foot }}^{-}-0\right)^{2}+Q_{T_{1}, 2}\left(\mu^{-}\right)^{2}
$$

where $Q_{T_{1}, 1}$ and $Q_{T_{1}, 2}$ are positive weights. The term $Q_{T_{1}, 1}\left(y_{\text {foot }}^{-}-0\right)^{2}$ penalizes the height of the foot from the ground $y=0$ to approximately encode the touch-down condition in a time-based formulation. Note that in order to minimize this term, it is important to find an appropriate flight time $T_{1}$ as the trajectory of the center of mass cannot be directly controlled in the flight phase for a given initial lift-off condition. The term $Q_{T_{2}, 2}\left(\mu^{-}\right)^{2}$ minimizes energy loss at touch-down where $\mu^{-}$is called the energy dissipation coefficient [27]

$\mu^{-}=\dot{x}_{\text {com }}^{-} \cos \theta^{-}+\dot{y}_{\text {com }}^{-} \sin \theta^{-}+r_{0} \dot{\theta}^{-}=\dot{x}_{\text {foot }}^{-} \cos \theta^{-}+\dot{y}_{\text {foot }}^{-} \sin \theta^{-}$

motivated from the study of passive running [27] to find an appropriate leg angle and foot velocity relative to the ground at touch-down. Note that if $\mu^{-}=0$, there is no energy loss at impact. In the running cost, we minimize the control effort $u_{1}$ and $u_{2}$ while small weights are used for penalizing the magnitude of stiffness $u_{3}$ and $u_{4}$ for regularization.

The initial conditions were obtained by choosing the desired initial horizontal velocity and lift-off angle of the leg $\left(\dot{x_{0}}, \theta_{0}\right)$ and computing the remaining parameters with the assumption of synchronization between the oscillatory movements of the leg swing and compression in the passive running model [26], [27]. In the following simulation, we use the robot parameters in [26].

3) Simulation Results: We choose the desired initial condition at lift off as $\dot{x}_{0}=2.0(\mathrm{~m})$ and $\theta_{0}=-6.0$ (deg). The rest is obtained based on an approximated condition of passive running [27] for the nominal model of [26]. Note that with this initial condition, the passive dynamics (no control) of the robot can achieve several steps of running as reported in [26], [27], however, eventually, it will fail since passive running is intrinsically unstable.

Using the proposed method, we simultaneously obtained the optimal feedback control for the control commands

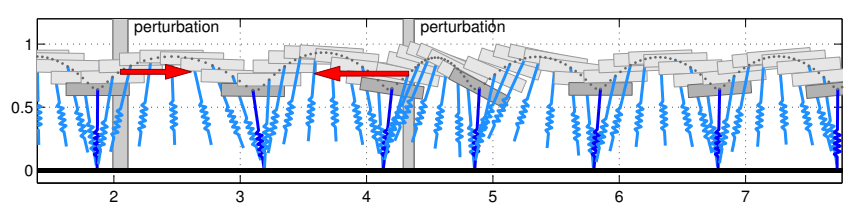

(a) Movement of the hopping robot horizontal velocity

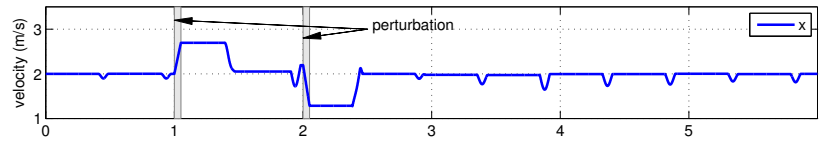
body height
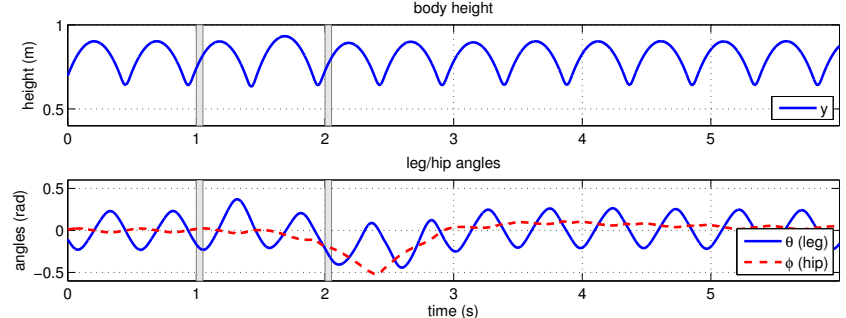

(b) Forward velocity, body height, joint angles

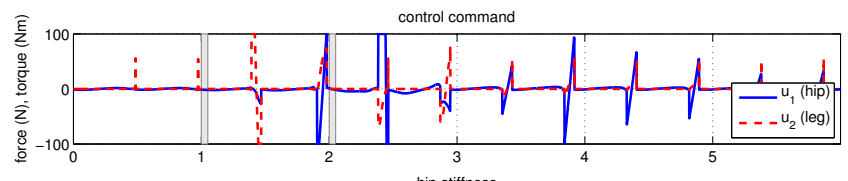

hip stiffness

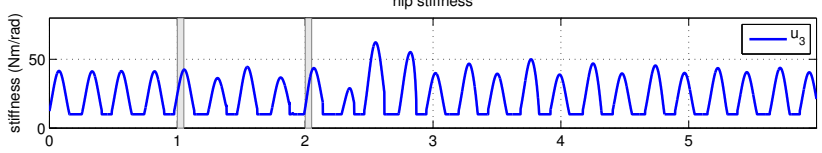
leg stiffness

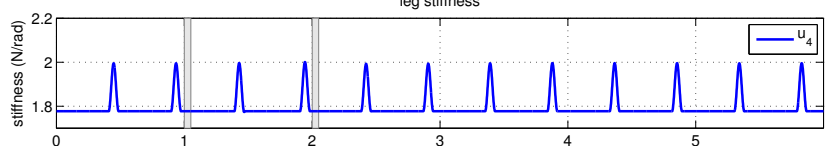

(c) Control commands, and hip and leg stiffness

Fig. 7. Application of the optimized controller to achieve steady state hopping with multiple cycles. External perturbations were applied in order to evaluate the robustness of the obtained optimal feedback control law. The robot was able to continue to run after the application of the perturbations without falling over.

$\left(u_{1}, u_{2}\right)$ and stiffness $\left(u_{3}, u_{4}\right)$, and found the flight time $T_{1}$ and the period for one complete cycle $T_{f}$ for one hopping cycle. The optimized flight time and one hopping cycle were $T_{1}=0.410(\mathrm{sec})$ and $T_{f}=0.487(\mathrm{sec})$. Since the obtained controller is based on an assumption of time-based switching, there could be some mismatch in the exact timing in the switching condition when applied to realistic event based switching dynamics (flight to stance at touch-down $y_{\text {foot }}=0$, stance to flight when $r=r_{0}$ ) to achieve multiple cycles of locomotion. One of the benefits of our approach is that it provides a locally optimal feedback control, deviations from the optimal trajectory can be corrected, which will be illustrated in the following examples.

a) Comparison to Individual Phase Optimization: As a comparison, we optimized the control command, stiffness and the movement duration in a sequential manner individually for the flight phase subsequently followed by the stance phase for one cycle of the movement. The optimized movement duration for the flight phase was $T_{1, \text { ind }}=0.410$ (sec) and for the stance phase was $T_{\text {stance, } i n d}=0.080$ (sec), i.e., the total duration was $T_{f, i n d}=0.490$ (sec). The total cost for this individual optimization was $J_{i n d}=$ 
1.686 which is comparable to the complete optimization case $J_{\text {comp }}=1.624$ mentioned above. The optimized trajectories, control commands and stiffness profiles are similar between these two cases. However, interestingly, there found notable difference in the robustness of the controller when these two were applied to the event based switching dynamics where the role of the feedback control becomes prominent.

The controller with complete cycle optimization was able to achieve continuous stable running over multiple cycles. However, with the controller obtained by individual optimization, the robot failed to continue to run after 25 steps of hopping. Although this is an empirical observation, it turned out that this difference presumably came from the difference in the optimal feedback gains. In the complete cycle optimization, the optimal feedback gains take the future goal until the end of the hopping cycle into account including both the flight and stance phases with the via-point and terminal costs. However, in the individual optimization, corrections are made only considering the immediate goal specified by the terminal cost in each phase. This result highlights the benefits of optimizing the whole cycle of the movement in comparison to individually optimizing the movement in a sequential manner. This comparison is reported in the accompanied video.

b) Robustness against Perturbations: In this simulation, we evaluate the robustness of the obtained optimal feedback controller by applying external perturbations while the robot is running. At $t=1.0(\mathrm{sec})$, the robot is pushed forward with $F_{x}=150(\mathrm{~N})$ and at $t=2.0(\mathrm{sec})$, a backward perturbation is applied $F_{x}=-250(\mathrm{~N})$ for the duration of 0.05 (sec), respectively. Fig. 7 (a) depicts the movement of the robot from $t=0.7 \sim 3.9$ (sec). Fig. 7 (b) show the forward velocity $\dot{x}$ (top), body height $y_{\text {com }}$ (middle), and leg angle $\theta$ and hip angle $\phi$ (bottom) from $t=0$ to $t=6$ (sec). Fig. 7 (c) show the control commands $u_{1}$ and $u_{2}$ (top), hip stiffness $u_{3}$ (center) and leg stiffness $u_{4}$ (bottom). The simulation result illustrate that the after the perturbations, the robot was able to stabilize the periodic running behavior without falling over demonstrating the robustness of the optimal feedback controller and the feasibility of the proposed approach in this problem setting.

\section{Conclusion}

In this paper, we have presented a systematic methodology for movement optimization with multiple phases and switching dynamics in robotic systems with variable stiffness actuation. Tasks including switching dynamics and interaction with an environment is approximately modelled as a hybrid dynamical system with time-based switching. With an appropriate choice of the composite cost function to encode the task, we have demonstrated the effectiveness of the proposed approach in numerical simulations. Future work will aim at hardware implementation of the proposed approach including variable damping [28].

\section{ACKNOWLEDGMENTS}

This work was funded by the EU Seventh Framework Programme (FP7) as a part of the TOMSY project.

\section{REFERENCES}

[1] R. Van Ham, et al., "Compliant actuator designs," IEEE Rob. and Aut. Mag., vol. 16, no. 3, pp. 81-94, 2009.

[2] D. Braun, M. Howard, and S. Vijayakumar, "Optimal variable stiffness control: formulation and application to explosive movement tasks," Autonomus Robots, vol. 33, no. 3, pp. 237-253, 2012.

[3] D. J. Braun, F. Petit, F. Huber, S. Haddadin, P. van der Smagt, A. AlbuSchäffer, and S. Vijayakumar, "Robots driven by compliant actuators: optimal control under actuation constraints," in IEEE Tras. on Robotics, vol. 29, no. 5, 2013.

[4] J. Nakanishi, K. Rawlik, and S. Vijayakumar, "Stiffness and temporal optimization in periodic movements: An optimal control approach," in IEEE/RSJ IROS, 2011.

[5] L. C. Visser, S. Stramigioli, and A. Bicchi, "Embodying desired behavior in variable stiffness actuators," in IFAC World Congress, 2011.

[6] J. Nakanishi and S. Vijayakumar, "Exploiting passive dynamics with variable stiffness actuation in robot brachiation," in $R: S S, 2012$.

[7] G. Bätz, et al., "Ball dribbling with an underactuated continuous-time control phase: Theory \& experiments," in IEEE/RSJ IROS, 2010.

[8] J. W. Grizzle, G. Abba, and F. Plestan, "Asymptotically stable walking for biped robots: Analysis via systems with impulse effects," IEEE Trans. on Automatic Control, vol. 46, no. 1, pp. 51-64, 2001.

[9] N. Rosa Jr., A. Barber, R. D. Gregg, and K. M. Lynch, "Stable openloop brachiation on a vertical wall," in IEEE ICRA, 2012.

[10] A. W. Long, T. D. Murphey, and K. M. Lynch, "Optimal motion planning for a class of hybrid dynamical systems with impacts," in IEEE ICRA, 2011.

[11] M. S. Branicky, V. S. Borkar, and S. K. Mitter, "A unified framework for hybrid control: Model and optimal control theory," IEEE Trans. on Automatic Control, vol. 43, no. 1, pp. 31-45, 1998.

[12] M. S. Shaikh and P. E. Caines, "On the hybrid optimal control problem: Theory and algorithms," IEEE Trans. on Automatic Control, vol. 52, no. 9, pp. 1587-1603, 2007.

[13] H. J. Sussmann, "A maximum principle for hybrid optimal control problems," in IEEE CDC, 1999.

[14] X. Xu and P. J. Antsaklis, "An Approach to General Switched Linear Quadratic Optimal Control Problems with State Jumps," in Int. Symp. on Math. Theory of Networks and Systems, 2002.

[15] X. Xu and P. J. Antsaklis, "Quadratic optimal control problems for hybrid linear autonomous systems with state jumps," in American Control Conf., 2003.

[16] A. E. Bryson and Y.-C. Ho, Applied Optimal Control. 1975.

[17] Y. Tassa, T. Erez, and E. Todorov, "Synthesis and stabilization of complex behaviors through online trajectory optimization," in IEEE/RSJ IROS, 2012.

[18] M. Posa and R. Tedrake, "Direct trajectory optimization of rigid body dynamical systems through contact," in International Workshop on the Algorithmic Foundations of Robotics, 2012.

[19] X. Xu and P. J. Antsaklis, "Optimal control of switched systems based on parameterization of the switching instants," IEEE Trans. on Automatic Control, vol. 49, no. 1, pp. 2-16, 2004.

[20] T. M. Caldwell and T. D. Murphey, "Single integration optimization of linear time-varying switched systems," in American Control Conf., 2011.

[21] M. Egerstedt, Y. Wardi, and F. Delmotte, "Optimal control of switching times in switched dynamical systems," in IEEE CDC, 2003.

[22] W. Li and E. Todorov, "Iterative linearization methods for approximately optimal control and estimation of non-linear stochastic system," Int. J. of Control, vol. 80, no. 9, pp. 1439-1453, 2007.

[23] T. M. Caldwell and T. D. Murphey, "Switching mode generation and optimal estimation with application to skid-steering," Automatica, vol. 47, no. 1, pp. 50-64, 2011.

[24] K. Rawlik, M. Toussaint, and S. Vijayakumar, "An approximate inference approach to temporal optimization in optimal control," in NIPS, 2010.

[25] R. V. Ham, et al., "MACCEPA, the mechanically adjustable compliance and controllable equilibrium position actuator: Design and implementation in a biped robot," Robotics and Autonomous Sys., vol. 55, no. 10, pp. 761-768, 2007.

[26] M. Ahmadi and M. Buehler, "Stable control of a simulated one-legged running robot with hip and leg compliance," IEEE Trans. on Rob. Aut., vol. 13, no. 1, pp. 96-104, 1997.

[27] S. Hyon and T. Emura, "Energy-preserving control of a passive onelegged running robot," Advanced Robotics, vol. 18, pp. 357-381, 2004

[28] A. Radulescu, M. Howard, D. J. Braun, and S. Vijayakumar, "Exploiting variable physical damping in rapid movement tasks," in IEEE/ASME AIM Conf., 2012. 